\title{
LXIII. On the calculations requisite for predicting occultations of stars by the moon
}

\section{Professor Bessel}

To cite this article: Professor Bessel (1829) LXIII. On the calculations requisite for predicting occultations of stars by the moon , Philosophical Magazine Series 2, 6:36, 410-416, DOI: $10.1080 / 14786442908675186$

To link to this article: http://dx.doi.org/10.1080/14786442908675186

里 Published online: 14 Jul 2009.

Submit your article to this journal $₫$

Џ Article views: 2

Q View related articles $\square$ 


\section{[ 410$]$}

LXIII. On the Calculations requisite for predicting Occultations of Stars by the Moon. By Professor BEsSEL.

[Concluded from p. 342.]

7. THE quantities referring to the place of observation, 1 "viz. $u, v, u^{\prime}, v$, depend on the height of the pole and the sidereal time at that place. It is unnecessary to calculate $\rho$ and $\phi^{\prime}$ separately, as we have

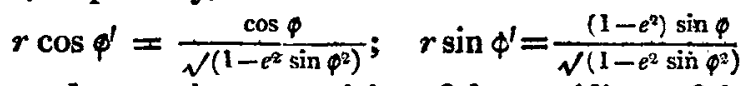

where $e$ denotes the eccentricity of the meridians of the earth. For Professor Encke's example, we have $\phi=52^{\circ} 31^{\prime} 15^{\prime \prime}$, and the oblateness of the earth $=\frac{1}{30278}$, hence

$$
\begin{aligned}
& \log \cdot r \cos \phi^{\prime}=9 \cdot 78505 \\
& \log \cdot r \sin \phi^{\prime}=9 \cdot 89752
\end{aligned}
$$

If we denote by $\mu^{\prime}$ the sidereal time, corresponding to the mean time $T$ expressed in degrees, \&c., the sidereal time corresponding to $\mathrm{T}+t$ will be

and consequently $\sin (\mu-\mathbf{A})=$

$$
\mu=\mu^{\prime}+t .54147^{\prime \prime} \cdot 84,
$$

$$
\begin{gathered}
\sin \left(\mu^{\prime}-\mathrm{A}\right)+2 \cdot \sin \left[t .27073^{\prime \prime} \cdot 92\right] \cdot \cos \left[\mu^{\prime}-\mathbf{A}+t .27073^{\prime \prime} \cdot 92\right] \\
\cos (\mu-\mathrm{A})= \\
\cos \left(\mu^{\prime}-\mathbf{A}\right)-2 \sin \left[t .27073^{\prime \prime} \cdot 92\right] \cdot \sin \left[\mu^{\prime}-\mathbf{A}+t .27073^{\prime \prime} \cdot 92\right]
\end{gathered}
$$

We have, therefore,

$u=r \cdot \cos \phi^{\prime} \cdot \sin \left(\mu^{\prime}-\mathrm{A}\right)$

$v=r \sin \phi^{\prime} \cdot \cos \mathrm{D}-r \cdot \cos \phi^{\prime} \sin \mathrm{D} \cos \left(\mu^{\prime}-\mathrm{A}\right)$

$u^{\prime}=r \cdot \cos \phi^{\prime} \cdot \frac{2 \sin \left[t .27073^{\prime \prime}{ }^{\prime 2}\right]}{t} \cos \left[\mu^{\prime}-\mathrm{A}+t .27073^{\prime \prime} \cdot 92\right]$

$v^{\prime}=r \cdot \cos \phi^{\prime} \sin \mathrm{D} . \frac{2 \sin \left[t .27073^{\prime \prime} \cdot 92\right]}{t} \sin \left[\mu^{\prime}-\mathbf{A}+t .27073^{\prime \prime} \cdot 92\right]$

For facilitating the calculation of $u^{\prime}$ and $v^{\prime}$ there is a Table at the end of this paper which contains the values of

$$
\log \frac{2 \sin \left[t .27073^{\prime \prime} \cdot 92\right]}{t}=\log \cdot \lambda
$$

and $\ldots . . t .27073^{11} \cdot 92=x$ for values of $t$ between 0 and $1 \cdot 5$.

8. I shall now finish the example above adduced. For $\mathrm{T}=7^{\mathrm{h}}$ the sidereal time will be $7^{\mathrm{h}} 54^{\prime} 7^{\prime \prime} \cdot 264$, and therefore

$$
\begin{aligned}
& \mu^{\prime}=118^{\circ} 31^{\prime} 49^{\prime \prime} \cdot 0 \\
& \mu^{\prime}-\mathrm{A}=-50 \quad 42 \quad 17 \cdot 6
\end{aligned}
$$

We obtain, therefore,

$$
u=-0.4 .7177 ; v=+0.75914
$$

and next

$$
\begin{gathered}
m \sin \mathrm{M}=p-u=-0 \cdot 19355 \\
m \cos \mathrm{M}=q-v=-0 \cdot 20674 \\
\mathrm{M}=223^{\circ} 6^{\prime} 46^{\prime \prime} ; \quad \log m=9 \cdot 45210 .
\end{gathered}
$$


Prof. Bessel on the Occultations of Stars by the Moon. 411

As a first approximation we assume the value of $t$ in the quantities $p^{\prime}, q^{\prime}, u^{\prime}, v^{\prime}=0$, and obtain

$$
\begin{array}{rlrl}
p^{\prime} & =+0.5240 & q^{\prime} & =-0.1679 \\
u^{\prime} & =+0.1013 & v^{\prime} & =-0.0091 \\
n \sin \mathrm{N} & =+0.4227 ; & n \cos \mathrm{N} & =-0.1588 \\
\mathrm{~N} & =110^{\circ} 35^{\prime} 26^{\prime \prime} ; & \log n & =9.65470 \\
t & =+0.2402 \mp 0.1690
\end{array}
$$

or, Immersion $7^{\text {h }} 07^{72}$; Emersion $7^{\text {h }} 4092$.

We next obtain for the second approximation by the formulæ of section 6 .

$$
\begin{aligned}
& \text { Immersion. Emersion. } \\
& p^{\prime} \ldots \ldots+0.52402+0.52404 \\
& q^{\prime} \ldots \ldots-0.16790 \quad-0 \cdot 16791
\end{aligned}
$$

and by the Table at the end of this paper,

$$
\begin{array}{cc}
x \ldots \ldots+32^{\prime} 7^{\prime \prime} \cdot 7 & +3^{\circ} 4^{\prime} 38^{\prime \prime} \cdot 6 \\
\log \lambda \ldots \ldots 9 \cdot 41915 & 9 \cdot 41895
\end{array}
$$

by which we shall find next

$$
\begin{array}{cc}
u^{\prime}=\ldots \ldots+0.10250 & +0.10780 \\
v^{\prime}=\ldots \ldots-0.00907 & -0.00873 \\
n \sin \mathrm{N}=+0.42152 & +0.41624 \\
n \cos \mathrm{N}=-0.15883 & -0.15918 \\
\mathrm{~N}=110^{\circ} 38^{\prime} 47^{\prime \prime} & 110^{\circ} 55^{\prime} 41^{\prime \prime} \\
\log n=9^{\circ} 65365 & 9.64898 \\
t= \begin{cases}+0.24026 & +0.23997 \\
-0.16857 & +0.16621\end{cases} & +0.40618
\end{array}
$$

The third approximation gives again the values of $p^{\prime}$ and $g^{\prime}$ obtained in the second approximation; and besides

$$
\begin{array}{cl}
x \ldots \ldots+32^{\prime} 20^{\prime \prime} \cdot 9 & +3^{\circ} 3^{\prime} 16^{\prime \prime} \cdot 9 \\
\log \lambda \ldots 9 \cdot 41915 & 9 \cdot 4.1895 \\
u^{\prime}=+0.10251 & +0.10774 \\
v^{\prime}=-0.00907 & -0.00873 \\
n \sin \mathrm{N}=+0.42151 & +0.41630 \\
n \cos \mathrm{N}=-0.15883 & -0.15918 \\
\mathrm{~N}=110^{\circ} 38^{\prime} 49^{\prime \prime} & 110^{\circ} 55^{\prime} 30^{\prime \prime} \\
\log n=9 \cdot 65364 & 9 \cdot 64904 \\
t=\left\{\begin{array}{l}
+0.24026 \\
-0.16857
\end{array}\right. & +0.23996 \\
=\frac{-0.16625}{+0.07169} & +0.40621
\end{array}
$$

and as these results differ very little from the preceding ones, we may here conclude the calculation. We have therefore the times of the two phænomena $=7^{\mathrm{b}} 4^{\prime} 18^{\prime \prime} \cdot 1$, and $7^{\mathrm{b}} 24^{\prime} 22^{\prime \prime} \cdot 4$, and the angle denoted by $\mathrm{Q}=36^{\circ} 49^{\prime} \cdot 6$, and $5^{\circ} 9^{\prime} \cdot 2$.

$$
3 \mathbf{G} 2
$$

9. Such 


\section{Prof. Bessel on the Calculations requisite for predicting}

9. - Such an accurate calculation is, however, not required when the circumstances of the occultation are only wanted for the purpose of making the observation; in that case an error of one minute is of no consequence; and if every thing requisite for this purpose only is wanted, it will be sufficient, provided $t$ falls below, or at least does not much exceed one hour* to apply the following much shorter calculation. In place of

$$
\frac{\cos \delta \cdot \sin (\alpha-A)}{\sin x} \text { and } \frac{\sin \delta \cdot \cos D-\cos \delta \cdot \sin D \cos (\alpha-A)}{\sin \pi}
$$

we put

$$
\frac{\alpha-A}{x} \cos \delta \quad \text { and } \frac{\delta-D}{\pi}
$$

and neglecting the variations of $\cos \delta$ and $\pi$, so as to designate the right ascension and declination of the moon at the time $T$ by $\alpha$ and $\delta$ and their hourly variations by $\Delta \alpha$ and $\Delta \hat{o}$, we have

$$
\begin{array}{ll}
p=\frac{a-A}{\pi} \cos \delta ; & p^{\prime}=\frac{\Delta \pi}{\pi} \cos \delta ; \\
q=\frac{\delta-D}{\pi} ; & q^{\prime}=-\frac{\Delta \delta}{\pi} .
\end{array}
$$

We next neglect $t$ in the expressions for $u^{\prime}$ and $v^{\prime}$ and obtain

$$
\begin{aligned}
& u=r \cos \phi^{\prime} \cdot \sin \left(\mu^{\prime}-\mathrm{A}\right) \\
& v=r \sin \phi^{\prime} \cdot \cos \mathrm{D}-r \cos \phi^{\prime} \cdot \sin \mathrm{D} \cdot \cos \left(\mu^{\prime}-\mathrm{A}\right) \\
& u^{\prime}=r \cos \phi^{\prime} \cdot \lambda \cos \left(\mu^{\prime}-\mathrm{A}\right) \\
& v^{\prime}=r \cos \phi^{\prime} \cdot \lambda \sin \left(\mu^{\prime}-\mathrm{A}\right) \sin \mathrm{D}
\end{aligned}
$$

For the place to which the calculations refer, the logarithms of $r \cos \phi^{l}$ and $r \sin \phi^{\prime}$ are to be considered as known. If we write $a$ for $r \cos \phi^{\prime} \sin \left(\mu^{\prime}-\mathbf{A}\right)$; $b$ for $r \cos \phi^{\prime} \cos \left(\mu^{\prime}-\Lambda\right)$; $c$ for $r \sin \phi^{\prime} \cos \mathrm{D}$, we have

$$
\begin{aligned}
& u=a ; \quad u^{\prime}=b . \lambda ; \quad v=c-b \sin \mathrm{D} \\
& v^{\prime}=a \cdot \lambda \sin \mathrm{D}: \text { where } \log \lambda=9^{\circ} 4192 \text { and } c \text { may }
\end{aligned}
$$

be taken from a small table which exhibits the value of this quantity for every degree of $D$ with sufficient accuracy to four figures of decimals. The solution of equation (6) remains the same. In order to have a clear view of the calculation, I subjoin here the whole detail of it:

$$
\begin{gathered}
\text { 1830, April 5. 82 Leonis. } \\
\mathrm{T}=7^{\mathrm{h}} ; \mu^{\prime}=118^{\circ} 32^{\prime} ; \mu^{\prime}-\mathrm{A}=-50^{\circ} 42^{\prime} ; \pi=54^{\prime} \cdot 18 \\
\alpha=168^{\circ} 37^{\prime} \cdot 93 ; \delta=44^{\prime} \cdot 00 ; \Delta \alpha=28^{\prime} \cdot 50 \\
\mathrm{~A}=\frac{16914^{\circ} \cdot 11}{-36 \cdot 18} \mathrm{D}=\frac{414 \cdot 08}{+29 \cdot 92} \Delta \delta=-9 \cdot 08
\end{gathered}
$$

* Should this supposition not be justified at the end of the calculation, or for other reasons a greater accuracy be desired, it may be obtained by a second approximation for $u^{\prime}$ and $v^{\prime}$ without changing $p^{\prime}$ and $q^{\prime}$. It is easily seen that the error of the first approximation increases with the distance of the star from the path of the moon's centre, and that its limits cannot be unconditionally assigned. 
$\cos \delta$

$$
\begin{aligned}
& l .(\alpha-\mathrm{A})=1.5585 n-0.0015 \mid \quad l \cdot r \cos \varphi^{\prime}=9.7850 \quad c=0.7876 \\
& \begin{array}{l}
l . \Delta \alpha=1.4548 \\
.(\partial-D)=1.4760
\end{array}-0.0015 \quad \begin{array}{l}
l \cdot \sin \left(\mu^{\prime}-\mathrm{A}\right)=9 \cdot 8887 n b \sin \mathrm{D}=0.0285 \\
l \cdot \cos \left(\mu^{\prime}-\mathrm{A}\right)=9.8017
\end{array} \\
& \text { l. } \Delta \delta=0.9481 n \\
& \text { l. } l \pi=\overline{8 \cdot 2662} \\
& \text { l. } a=\overline{9 \cdot 6737 n} \\
& l . b=9.5867 \\
& l . \lambda=\overline{9.4192} \\
& l . \sin \mathrm{D}=8 \cdot 8683 \\
& p=-0.6656 \quad u=-0.4717 \quad l . m \sin \mathrm{M}=9.2876 n \\
& q=+0.5523 \quad v=\$ 0.7591 \quad \text { l. } m \cos \mathrm{M}=9.3156 n \\
& p^{\prime}=+0.5242 \quad u^{\prime}=+0.1014 \quad l . n \sin \mathrm{N}=9.6261 \\
& q^{\prime}=-0 \cdot 1638 \quad v^{\prime}=-0.0092 \quad \text { l. } n \cos \mathrm{N}=9 \cdot 1892 n \\
& M=223^{\circ} 9^{\prime} ; l m=9.4525 ; \mathrm{N}=110^{\circ} 5^{l} ; l n=9 \cdot 6533 \\
& l . \frac{m}{k}=0.0171 \quad l .-\frac{m}{n}=9 \cdot 7992 n \quad l . \frac{k}{n}=9 \cdot 7821
\end{aligned}
$$

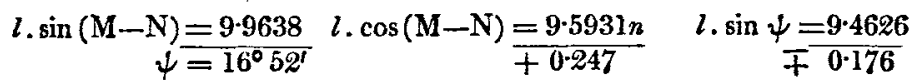

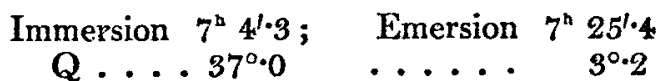

10. The method of calculating which Mr. Encke has followed in constructing his Ephemeris for 1820, is perhaps as easy as the one here given; but it requires the previous calculation of some tables. The use of such tables not being required for the method here given, it will be easy for observers in other places to calculate every occultation for their horizons if the values of $p, q, p^{\prime}, q^{\prime}$ and the hour-angle of the star at the time $T$, viz. $\mu^{\prime}-A$, which I will now denote by $h$, are given.

These data referring to the moon alone remain unchanged in the calculations for other places; only $h$ is to be changed into $h+d$, where the positive $d$ denotes eastern longitude from Berlin. For such a place we have

$$
\begin{gathered}
a=r \cos \varphi^{\prime} \sin (h+d) \\
b=r \cos \phi^{\prime} \cos (h+d) \\
u=a ; \quad u^{\prime}=b \cdot \lambda \\
v=c-b \cdot \sin \mathrm{D}, \quad v^{\prime}=a \cdot \lambda \sin \mathrm{D} ;
\end{gathered}
$$

hence $m, \mathrm{M}, n, \mathrm{~N}$, and lastly, the two values of $t$, which will show the mean time at Berlin, at the two moments in which the disappearance and reappearance will happen at the place for which the calculation has been made. This process will likewise be illustrated by calculating the occultation of 82 Leonis for Altona.

We assume as given :

$$
\mathrm{T}=7^{\mathrm{h}}\left\{\begin{array}{c}
p=-0.6656, p^{\prime}=+0.5242 \\
q=+0.5523, q^{\prime}=-0.1638
\end{array}\right\} h=-50^{\circ} 42^{\prime}
$$


414 Prof. Bessel on the Calculations requisite for predicting For Altona we have
l. $r \cos \varphi^{\prime}=9 \cdot 77485$,
$l . r \sin \phi^{\prime}=9 \cdot 90949, \quad d=-3^{\circ} 27^{\prime}$

l. $r \cos \phi^{\prime}=9 \cdot 7749$

l. $\sin (h+d)=9 \cdot 9088 n$

l. $\cos (h+d)=9 \cdot 7677$

l. $a \equiv 9 \cdot 6837 n$

$l . b=9 \cdot 5426$

$$
\begin{array}{ll}
u=-0.4827 & c \\
v=+0.7728 & b \sin \mathrm{D}=0.7986 \\
u^{\prime}=+0.0916 & \\
v^{\prime} \neq-0.0094 &
\end{array}
$$

l. $\lambda=9.4 .192$

$l . \sin \mathrm{D}=8.8683$

$l . m \sin \mathrm{M}=9 \cdot 2622 n \quad \mathrm{M}=219^{\circ} 40^{\prime}, \quad l . m=9 \cdot 4571$

$l, m \cos \mathrm{M}=9 \cdot 34.34 n$

$l . n \sin \mathrm{N}=9.6361 \quad \mathrm{~N}=109^{\circ} 39^{\prime}, \quad l . n=9.6621$

$l \cdot n \cos \mathrm{N}=9 \cdot 1887 n$

$$
\begin{aligned}
& l . \frac{m}{k}=0.0217 \quad \text { l. } \frac{m}{n}=9.7950 n \quad l . \frac{k}{n}=9.7733
\end{aligned}
$$

\begin{tabular}{|c|c|c|c|c|c|c|}
\hline$t$ & $\log \lambda$ & & $x$ & $t$ & $\log \lambda$ & $x$ \\
\hline $0 \cdot 00$ & $9 \cdot 41916$ & $\stackrel{0}{0}$ & $\begin{array}{l}o \\
0\end{array}$ & $0 \cdot 19$ & $9 \cdot 41911$ & $I_{25} 44^{\prime \prime} 1$ \\
\hline 01 & 916 & 04 & $430 \cdot 7$ & 0.20 & 911 & $\begin{array}{lll}1 & 30 & 14.8\end{array}$ \\
\hline 0.02 & 916 & $0 \mathrm{~s}$ & $9 \quad l \cdot 5$ & 0.21 & 910 & 13445.5 \\
\hline 0.03 & 915 & 015 & $332 \cdot 3$ & 0.22 & 910 & $\begin{array}{llll}1 & 39 & 16 \cdot 3\end{array}$ \\
\hline 0.04 & 915 & 018 & $8 \quad 3.0$ & 0.23 & 909 & $14347 \times 0$ \\
\hline 0.05 & 915 & 022 & $233 \cdot 7$ & 0.24 & 908 & $148 \quad 17 \cdot 7$ \\
\hline 0.06 & 915 & 027 & $7 \quad 4 \cdot 4$ & 0.25 & 908 & 15248.5 \\
\hline 0.07 & 915 & 031 & 135.2 & 0.26 & 907 & $\begin{array}{lll}1 & 57 & 19 \cdot 2\end{array}$ \\
\hline 0.08 & 915 & 036 & $\begin{array}{ll}6 & 5 \cdot 9\end{array}$ & 0.27 & 907 & $2 \quad 150 \cdot 0$ \\
\hline $0 \cdot 09$ & 915 & 040 & 036.7 & 0.28 & 906 & 2620.7 \\
\hline $0 \cdot 10$ & 914 & 045 & $7 \cdot 4$ & 0.99 & 903 & $210.51 \cdot 4$ \\
\hline 0 & 914 & 045 & $938 \cdot 1$ & 0.30 & 904 & 21522,2 \\
\hline $0 \cdot 12$ & 914 & 054 & $4 \quad 8 \cdot 9$ & 0.31 & 904 & $\begin{array}{lll}2 & 19 & 52-9\end{array}$ \\
\hline $0 \cdot 13$ & 913 & 058 & $\begin{array}{ll}839 \cdot 6\end{array}$ & 0.32 & 903 & $22423 \cdot 7$ \\
\hline 0.14 & 9.13 & $1 s$ & $\begin{array}{ll}3 & 10 \cdot 4\end{array}$ & 0.53 & 902 & $22854 \cdot 4$ \\
\hline $0 \cdot 1$ & 913 & 17 & $741 \cdot 1$ & 0.34 & 901 & $\begin{array}{llll}2 & 25 \cdot 1\end{array}$ \\
\hline 0 & 912 & 112 & $\begin{array}{lll}2 & 11 \cdot 8\end{array}$ & 0.35 & 900 & 23755.9 \\
\hline 0.17 & 912 & 116 & $642 \cdot 6$ & 0.96 & 899 & $24226 \cdot 6$ \\
\hline 0.18 & 912 & 121 & $113 \cdot 3$ & 0.37 & 899 & $24657 \cdot 4$ \\
\hline
\end{tabular}

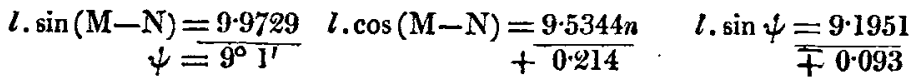

Immersion $7^{\mathrm{h}} 7^{\prime} \cdot 3$; Emersion $7^{\mathrm{h}} 18^{\mathrm{l}} \cdot 4^{\text {. }}$ Berlin time.

$$
\begin{array}{lll} 
& =653 \cdot 5 & \ldots \ldots 7 \\
\mathbf{Q} & =28^{\circ} \cdot 7 & \ldots \ldots 10^{\circ} \cdot 6
\end{array}
$$

Auxiliary TaBLE for the Calculations requisite for predicting Occultations of Stars.

Table 
Occultations of Stars by the Moon.

Table continued.

\begin{tabular}{|c|c|c|c|c|c|}
\hline$t$ & $\log \lambda$ & $x$ & $t$ & $\log \lambda$ & $x$ \\
\hline 0.38 & $9 \cdot 41898$ & $\stackrel{\circ}{2} 51^{\prime} 28{ }^{\prime \prime} 1$ & 0.81 & 9.41834 & $\begin{array}{lll}6 & \dot{5} & 299^{\prime \prime} \cdot 9\end{array}$ \\
\hline 0.39 & 897 & 25558.8 & 0.82 & 832 & $\begin{array}{lll}6 & 10 & 0.6\end{array}$ \\
\hline $0 \cdot 40$ & 896 & $\begin{array}{llll}3 & 0 & 29 \cdot 6\end{array}$ & 0.83 & 830 & $61431 \cdot 4$ \\
\hline 0.41 & 895 & $\begin{array}{lll}3 & 5 & 0.3\end{array}$ & 0.84 & 828 & $\begin{array}{lll}6 & 19 & 2 \cdot 1\end{array}$ \\
\hline 0.42 & 894 & $\begin{array}{lll}3 & 9 & 31 \cdot 1\end{array}$ & 0.85 & 825 & $\begin{array}{lll}6 & 23 & 32 \cdot 8\end{array}$ \\
\hline 0.43 & 893 & $\begin{array}{lll}3 & 14 & 1.8\end{array}$ & $0 \cdot 86$ & 823 & $\begin{array}{lll}6 & 28 & 3 \cdot 6\end{array}$ \\
\hline 0.44 & 891 & $\begin{array}{lll}3 & 18 & 32.5\end{array}$ & 0.87 & 821 & $632 \quad 34 \cdot 3$ \\
\hline 0.45 & 890 & $\begin{array}{lll}323 & 3 \cdot 3\end{array}$ & 0.88 & 819 & 6.375 .1 \\
\hline $0 \cdot 46$ & 889 & $\begin{array}{lll}327 & 340\end{array}$ & $0 \cdot 89$ & 817 & $\begin{array}{llll}6 & 4.1 & 35 \cdot 8\end{array}$ \\
\hline 0.47 & 888 & $\begin{array}{lll}3 & 32 & 4 \cdot 8\end{array}$ & 0.90 & 815 & $646 \quad 6.5$ \\
\hline 0.48 & 887 & $\begin{array}{lll}3 & 35 \cdot 5\end{array}$ & 0.91 & 812 & $\begin{array}{llll}6 & 50 & 37 \cdot 3\end{array}$ \\
\hline 0.49 & 885 & $\begin{array}{llll}3 & 41 & 6 \cdot 2\end{array}$ & 0.92 & 810 & $\begin{array}{lll}6 & 55 & 8 \cdot 0\end{array}$ \\
\hline $0 \cdot 50$ & 884 & $\begin{array}{lll}345 & 37 \cdot 0\end{array}$ & 0.93 & 808 & $\begin{array}{llll}6 & 59 & 38.8\end{array}$ \\
\hline 0.51 & 883 & $\begin{array}{lll}350 & 7 \cdot 7\end{array}$ & 0.94 & 805 & $\begin{array}{lll}7 & 4 & 9 \cdot 5\end{array}$ \\
\hline 0.52 & 882 & $354 \quad 38 \cdot 4$ & 0.95 & 803 & $\begin{array}{llll}7 & 8 & 40 & 2\end{array}$ \\
\hline 0.53 & 881 & $\begin{array}{lll}3 & 59 & 9^{\bullet} 2\end{array}$ & 0.96 & 801 & 7131110 \\
\hline 0.54 & 879 & $4 \quad 339 \cdot 9$ & 0.97 & 798 & $71741 \cdot 7$ \\
\hline 0.55 & 878 & $\begin{array}{lll}4 & 8 & 10.7\end{array}$ & $0 \cdot 98$ & 796 & 72212.5 \\
\hline 0.56 & 876 & $41241 \cdot 4$ & 0.99 & 793 & $7 \& 643 \cdot 2$ \\
\hline 0.57 & 875 & $\begin{array}{lll}4 & 17 & 12 \cdot 1\end{array}$ & $1 \cdot 00$ & 791 & $\begin{array}{llll}7313 & 31\end{array}$ \\
\hline 0.58 & 874 & $4 \quad 2142 \cdot 9$ & 1.01 & 788 & $\begin{array}{lll}735 & 44^{\circ} 7\end{array}$ \\
\hline 0.59 & 872 & $\begin{array}{lll}4 & 26 & 13 \cdot 6\end{array}$ & $1 \cdot 02$ & 786 & $740 \quad 15^{\circ} 4$ \\
\hline 0.60 & 871 & $43044 \cdot 4$ & $1 \cdot 03$ & 783 & $74446^{\circ} 1$ \\
\hline 0.61 & 869 & $435 \quad 15 \cdot 1$ & 1.04 & 781 & $\begin{array}{lll}7 & 49 & 16\end{array}$ \\
\hline $0 \cdot 62$ & 868 & $\begin{array}{lll}49 & 45 \cdot 8\end{array}$ & 1.05 & 778 & $75347^{\circ} 6$ \\
\hline 0.63 & 866 & $\begin{array}{lll}4 & 44 & 16\end{array}$ & $1 \cdot 06$ & 775 & $758 \quad 18^{\circ} 4$ \\
\hline 0.64 & 865 & $\begin{array}{lll}4 & 47 \cdot 3\end{array}$ & $1 \cdot 07$ & 773 & $\begin{array}{lll}8 & 2 & 49 \\
1\end{array}$ \\
\hline 0.65 & 863 & $\begin{array}{lll}453 & 18 \cdot 1\end{array}$ & 1.08 & 770 & $\begin{array}{lll}8 & 7 & 19.8\end{array}$ \\
\hline $0 \cdot 66$ & 861 & $45748^{\circ} 8$ & $1 \cdot 09$ & 767 & $81150^{\circ} 6$ \\
\hline 0.67 & 860 & $\begin{array}{lll}5 & 2 & 19.5\end{array}$ & $1 \cdot 10$ & 765 & $\begin{array}{llll}8 & 16 & 21.3\end{array}$ \\
\hline 0.68 & 858 & $\begin{array}{lll}5 & 6 & 50: 3\end{array}$ & $1 \cdot 11$ & 762 & $82052 \cdot 1$ \\
\hline 0.69 & 856 & $\begin{array}{llll}5 & 11 & 21.0\end{array}$ & $1 \cdot 12$ & 759 & $\begin{array}{lll}8 & 25 & 22 \cdot 8\end{array}$ \\
\hline 0.70 & 854 & $\begin{array}{llll}5 & 15 & 51 \cdot 7\end{array}$ & 1.13 & 756 & $829 \quad 5.3 .5$ \\
\hline 0.71 & 8.53 & $\begin{array}{lll}5 & 202 & 925\end{array}$ & $1 \cdot 14$ & 753 & $83424^{\circ} 3$ \\
\hline 0.72 & 851 & $\begin{array}{llll}5 & 243 \bullet 2\end{array}$ & $1 \cdot 15$ & 751 & $83855^{\circ} 0$ \\
\hline 0.73 & 849 & $\begin{array}{lll}5 & 29 & 24 \cdot 0\end{array}$ & $1 \cdot 16$ & 748 & $\begin{array}{lll}8 & 45 & 25^{\circ}\end{array}$ \\
\hline 0.74 & 847 & 53354.7 & $1 \cdot 17$ & 745 & 84756.5 \\
\hline 0.75 & 845 & $\begin{array}{lll}5 & 38 & 25 \cdot 4\end{array}$ & $1 \cdot 18$ & 742 & $8 \quad 52 \quad 27^{\circ} 2$ \\
\hline 0.76 & 844 & $\begin{array}{lll}5 & 42 & 56 \cdot 2\end{array}$ & $1 \cdot 19$ & 739 & $85658 \%$ \\
\hline 0.77 & 842 & $\begin{array}{lll}5 & 47 & 26 \cdot 9\end{array}$ & $1 \cdot 20$ & 736 & $\begin{array}{lll}9 & 1 & 28.7\end{array}$ \\
\hline 0.78 & 840 & $55157 \cdot 7$ & $1 \cdot 21$ & 733 & $\begin{array}{lll}9 & 5 & 59.5\end{array}$ \\
\hline 0.79 & 838 & $\begin{array}{llll}5 & 56 & 28 \cdot 4\end{array}$ & $1 \cdot 22$ & 730 & $\begin{array}{llll}9 & 10 & 30 \cdot 2\end{array}$ \\
\hline 0.80 & 836 & $\begin{array}{llll}6 & 0 & 59 \cdot 1\end{array}$ & 1.23 & 727 & $\begin{array}{lll}9 & 15 & 0.9\end{array}$ \\
\hline
\end{tabular}

Table 
Table concluded.

\begin{tabular}{|c|c|c|c|c|c|c|c|c|}
\hline$t$ & $\log \lambda$ & \multicolumn{3}{|c|}{$\dot{*}$} & $t$ & $\log \lambda$ & $x$ & \\
\hline $1 \cdot 24$ & $9 \cdot 41724$ & & & $31 \cdot 7$ & $1 \cdot 38$ & $9 \cdot 41678$ & $10^{\circ} \dot{2} \dot{z}$ & $4 \stackrel{2}{2} \cdot 0$ \\
\hline 1.25 & 721 & 9 & 24 & $2 \cdot 4$ & 1.39 & 674 & 1027 & $12 \cdot 8$ \\
\hline $1 \cdot 26$ & 717 & 9 & 28 & $33 \cdot 2$ & $1 \cdot 40$ & 671 & 1031 & $43 \cdot 5$ \\
\hline $1 \cdot 27$ & 714 & 9 & 33 & $3 \cdot 9$ & $1 \cdot 41$ & 667 & 1036 & $14 \cdot \mathcal{2}$ \\
\hline $1 \cdot 28$ & 711 & 9 & 37 & $34 \cdot 6$ & $1 \cdot 42$ & 664 & 1040 & $45 \cdot 0$ \\
\hline $1 \cdot 29$ & 708 & & 42 & $5 \cdot 4$ & $1 \cdot 43$ & 660 & 1045 & $15 \cdot 7$ \\
\hline $1 \cdot 30$ & 705 & & 46 & $36 \cdot 1$ & $1 \cdot 44$ & 6.57 & 1049 & $46 \cdot 5$ \\
\hline $1 \cdot 31$ & 701 & 9 & 51 & $6 \cdot 8$ & $1 \cdot 45$ & 653 & 1054 & $17 \cdot 2$ \\
\hline $1 \cdot 32$ & 698 & 9 & 55 & $37 \cdot 6$ & $1 \cdot 46$ & 649 & 1058 & $47 \cdot 9$ \\
\hline 1.33 & 695 & 10 & 0 & $8 \cdot 3$ & $1 \cdot 47$ & 646 & 113 & $18 \cdot 7$ \\
\hline $1 \cdot 34$ & 691 & 10 & 4 & $39 \cdot 1$ & $1 \cdot 48$ & 642 & 117 & $49 \cdot 4$ \\
\hline $1 \cdot 35$ & 688 & & 9 & $9 \cdot 8$ & $1 \cdot 49$ & 638 & 1112 & $20 \cdot 2$ \\
\hline $1 \cdot 36$ & 685 & 10 & 13 & $40 \cdot 5$ & $1 \cdot 50$ & 635 & 1116 & $50 \cdot 9$ \\
\hline $1 \cdot 37$ & 681 & 10 & I 8 & $11 \cdot 3$ & & & & \\
\hline
\end{tabular}

F. W. BESSEL.

LXIV. On some Optical Phanomena. By Dr. J. Stokes*.

$\mathrm{A}_{\mathrm{S}}$ facts are the groundwork of science, and the commu-

nication of facts to those that love it the only means of its advancement, I shall give a detail of several optical phænomena of very rare occurrence, and also of some experiments on the transmission of light through very small boles.

On the 25th of August in this year, between 7 and 8 A.M., I witnessed the following:

There was a westerly wind, and the sky was thickly strewed with light fleecy clouds, so small as to resemble flocculi, and so numerous as to form a continuous layer. These were moving with great rapidity towards the sun. In fig. 1, let the point $a$ represent the zenith, and $a a^{\prime \prime}$ a line passing from thence to the sun. This line appeared to me to pass through the points of contact that four arches $b c, d e, f g, h i$ made with one another. They were rather faint, but exhibited prismatic colours; those of $b c$ being the most perfect, those of $h i$ the least: $f g$ cannot be properly called an arch. Its form possessed rather a striking analogy to that of a well-known curve; $b c$ appeared to be an $\operatorname{arc}$ of $60^{\circ}$ to a circle whose radius was in the zenith $a$, and its chord I think parallel to the horizon; its two extremities $b$ and $c$ were well defined : but those of $d e$ were not so; one of them, $d$, extending indefinitely on that side.

* Communicated by the Author. 